\title{
Spectrum of Synovial Sarcoma-clinicopathological and Immunohistochemical Correlation
}

\author{
Sunandalakshmi GV ${ }^{1}$, Sujitha Chougani ${ }^{2 *}$, Durga Kharidehal ${ }^{1}$, Ravi Sankar $V^{3}$ and Santhi Vissa ${ }^{1}$ \\ ${ }^{1}$ Department of pathology, Narayana Medical College, Nellore, Andhra Pradesh, India \\ ${ }^{2}$ Department of pathology, Yashoda Hospital, Secunderabad, Telangana, India \\ ${ }^{3}$ Department of Emergency Medicine, Narayana Medical College, Nellore, Andhra Pradesh. India
}

\section{ABSTRACT}

Background: Synovial sarcoma is the common adult soft tissue sarcomas affects the lower extremities. In poor resource settings, characterization by molecular methods may not be feasible always. This study is aimed to evaluate Sensitivity and Specificity of Transducinlike enhancer of split 1 (TLE1) immunohistochemical expression in Synovial sarcomas (SS) and its histological mimics and to study the clinicopathological features of Synovial sarcoma.

Method: A prospective study of two years duration from September 2013 to September 2015 conducted at the Department of Pathology, MNJ Institute of Oncology and Regional Cancer Centre, Redhills, Hyderabad. Total number of Synovial sarcomas was 30 . All original H \& E section of the tumor are reviewed and Immunohistochemistry (IHC) analysis was carried out on TLE1 mouse monoclonal antibody (TMA) blocks using TLE1 Antibody.

Results: The Median age was 30 years. The most common site of involvement was lower limbs. The most common mode of presentation was painless swelling. The most common variant of Synovial sarcoma was monophasic Spindle in 19 cases. Total number of TLE1 positive cases of Synovial sarcoma was $93.3 \%$. Total number of SS mimics showing TLE1 positivity was30\%. TLE1 showed Sensitivity $93.3 \%$ and Specificity- $73.3 \%$. In the present study negative predictive value is $91.6 \%$ and the positive predictive value is $77.7 \%$.

Conclusion: All small round blue cell tumors should be kept in mind while diagnosing poorly differentiated SS. TLE1 is a very sensitive marker for synovial sarcoma.TLE 1 is a highly sensitive marker for synovial sarcoma, but is less specific because of its positive expression in other mesenchymal tumors.

Keywords: Synovial Sarcoma, Ewing Sarcoma, Schwannoma, Vimentin

\section{Introduction}

Synovial sarcoma (SS) is a mesenchymal tumor, which displays a variable degree of epithelial differentiation including gland formation, and has a specific chromosomal translocation $\mathrm{t}(\mathrm{X} ; 18)(\mathrm{p} 11 ; \mathrm{q} 11)$ that leads to formation of a SS18-SSX fusion gene..$^{[1,2]}$ It is the fourth most common high grade soft tissue sarcoma, after Malignant fibrous histiocytoma, leiomyosarcoma, Rhabdomyosarcoma. It accounts for $5-10 \%$ of all soft tissue sarcomas and is accompanied by an often poor prognosis with high chance of metastasis despite surgical resection. Local control requires wide local excision and radiation therapy, causing significant morbidity in relatively young patients.

SS is most prevalent in adolescents and young adults aged between 15 - 40 years. Males are more often affected than females with Male: Female of $1.2: 1$.

Synovial sarcoma is a well characterised malignant soft tissue sarcoma that often occurs in close proximity to large joints of extremities. SS are known to occur at various sites.
Histologically, synovial sarcoma is subtyped into Biphasic SS, Monophasic SS, Poorly differentiated SS and Calcifying type ${ }^{[3]}$ The diagnosis of biphasic synovial sarcoma is generally straightforward, but the diagnosis of Monophasic SS and Poorly differentiated SS is challenging, because monophasic SS mimics most of the spindle cell lesions and poorly differentiated SS mimics small round blue cell tumors.

The diagnostic gold standard for Synovial sarcoma is demonstration of the characteristic translocation between the SS18 (SYT) gene on chromosome 18 and one of three SSX genes (SSX1, SSX2, or rarely SSX4) on chromosome $\mathrm{X}\left(\mathrm{t}(\mathrm{X} ; 18)(\mathrm{p} 11.2 ; \mathrm{q} 11.2)^{[4]}\right.$. Detection of $\mathrm{t}(\mathrm{X} ; 18)$ can be accomplished by cytogenetic karyotyping, fluorescence in situ hybridization (FISH) or reverse-transcriptase polymerase chain reaction (RT-PCR). The use of these techniques is limited by many practical issues (cost, specialized equipment, availability). Thus, in practice the diagnosis of Synovial sarcoma is usually based on histological examination and immunohistochemistry. 
Cytokeratin, EMA, CD99 and Bcl-2 are used to identify Synovial sarcomas, but have limited specificity and sensitivity. Transducin - like enhancer of split 1 (TLE 1) gene is a member of the TLE gene family and involved in control of hematopoiesis, neuronal, and terminal epithelial differentiation ${ }^{[5]}$ TLE 1 competes with $\beta$-catenin, which plays an important role in Wnt/ $\beta$-catenin signalling pathway. ${ }^{[6]}$ Several studies analyzed TLE1 as a diagnostic immunohistochemical marker for synovial sarcoma. ${ }^{[7,8]}$ In the present study, we evaluate immunohistochemical expression of TLE1 in Synovial sarcoma and its histologic mimics by tissue microarray technique. Aim of the study was to assess the utility of TLE1 as a diagnostic marker in Synovial sarcoma and to evaluate Sensitivity and Specificity of TLE1 immunohistochemical expression in Synovial sarcoma and its histological mimics.

Materials and methods:

A prospective study was conducted for a period of two years from September 2013 to September 2015.This study was conducted at the department of Pathology, MNJ Institute of Oncology and Regional Cancer Centre, Redhills, Hyderabad. The study was approved by the institutional ethics committee. The study data collected from the records from clinical diagnostics at the institute.

Inclusion Criteria: All cases which were diagnoses as synovial sarcoma and cases of mesenchymal neoplasms mimicking synovial sarcoma were included in the study.

Exclusion Criteria: Swellings presented as inflammatory lesions, with scanty or no cells on aspiration, patients who denied for a valid consent were excluded from the study

\section{Methodology}

Due importance was given to record a brief clinical history with age, Inpatient registration number, biopsy number, presenting symptoms and signs, CT and MRI findings. Trucut biopsy or core needle biopsy of the growth was done followed by wide excision. The specimens were received in 10\% formalin. Measurements of the specimens were recorded. Thorough gross examination was carried out and salient features like haemorrhage, necrosis, calcification and cyst formation were recorded. Parallel transverse sections were given through each half, about $1 \mathrm{~cm}$ apart. Depending on the size of tumour, adequate number of blocks was given. H\&E staining was done. IHC was carried out on whole sections using CD99, EMA, $\mathrm{Bcl}$, CK and all other markers which aid in differentiating SS from its mimics.

Tissue Microarray Preparation: The original H\&E section of the tumor are reviewed, representative areas were identified and marked on the corresponding tissue blocks. Care was taken, to avoid the area with necrosis and or hemorrhage. The tissue cores were extracted from the marked area and transferred into corresponding tissue block. Marked tissues were extracted from the donor block using Quick-Ray needle with $5 \mathrm{~mm}$ tip. One core was taken per case subject to availability of material. Tissue cores were delivered into corresponding holes of the recipient block and each core was numbered appropriately. Recipient block was put in embedding mold with cutting section faced down and incubated at $60^{\circ} \mathrm{C}$ for 30 minutes. Then, recipient block was embedded; sections were cut at $4 \mu \mathrm{m}$ thickness and taken on to charged slides for H\&E and for IHC studies.

Immunohistochemistry: IHC analysis was carried out on TMA blocks using TLE1 (1F5) Mouse Monoclonal Antibody (CELL MARQUE, USA). Concentrated working solution was diluted in 1 in 30 dilutions.

Immunohistochemistry Technique: The IHC was performed by semi-automated immunostainer (i6000, Biogenex). Immunohistochemistry (IHC) was done by Poly HRP technique. To fix, the sections were kept at $60^{\circ} \mathrm{c}$ for $30 \mathrm{~min}$. Dewaxing was done in 3 changes of xylene followed by hydration in graded alcohol and water. Antigen retrieval was done by using pressure cooker in TRIS EDTA at $\mathrm{pH}$ 9.0. After cooling to room temperature, sections were washed with three changes of distilled water to replace TRIS EDTA gradually. Then the slides were rinsed in phosphate buffer saline thrice for 5 minutes each followed by immersion in 3\% methanol $\mathrm{H} 202$ for 10 min to block endogenous peroxidase activity and treated with power block for 10 minutes to inhibit binding to non-specificsites. Sections were incubated with primary antibody (TLE 1) for $90 \mathrm{~min}$, then secondary antibody (super enhancer) for 20min and HRP-polymer for 30min. In between each of these above steps two rinses of PBS for 5 minutes each were applied. The antigen-antibody complex was visualized by using DAB (diaminobenxidine) as chromogen for $7 \mathrm{~min}$. Sections were counterstained with Harris' hemotoxylin for $1 \mathrm{~min}$. Then they were dehydrated through alcohol, cleared in xylene and mounted in DPX.

Positive control: Two cases showing SS18 break-apart by fluorescent in situ hybridization and showing positive staining with TLE 1 served as positive control.

Interpretation on IHC results: Nuclear immunoreactivity was graded as $0,1+, 2+$ and $3+$ based on intensity and percentage of cells.

More than $50 \%$ of the cells exhibit intense positivity which is visible with a $4 \mathrm{X}$ objective, was graded as $3+; 25-50 \%$ of the cells exhibiting intense positivity or more than $50 \%$ 
of the cells showing moderate intensity which is visible on $10 \mathrm{X}$ objective was graded as $2+; 5-25 \%$ of the cells with intense positivity graded as $1+$; less than $5 \%$ staining of tumour nuclei is graded as nil (0).

Statistical Analysis: The categorical variables were expressed as percentages, continuous variables as mean \pm standard deviation. The data was entered in Microsoft Excel sheet and TLE1 Expression was graded in to Grade 0, Grade 1, Grade 2 and Grade 3. Over all Sensitivity, Specificity, Positive Predictive value and Negative Predictive value of TLE 1were calculated using Microsoft Excel sheet.

\section{Results}

The study includes 30 cases of synovial sarcoma, 24 cases of monophasic synovial sarcoma mimics (2 Leiomyosarcoma, 4 Gastrointestinal stromal tumour, 2 Hemangiopericytoma, 3 Dermato fibrosarcomaprotuberans, 3 Fibrosarcoma, 3 MPNST, 2 Solitary fibrous tumour, 1 Malignant fibrous histiocytoma and 4 Schwannoma) and 6 cases of poorly differentiated sarcoma mimics (4 Ewing sarcomas and 1 Rhabdomyosarcoma, 1 Desmoplastic Small round cell tumor).

Maximum No. of Synovial sarcoma cases - 16 cases (64\%) belong to the age group between 20-50 years. Age range was 10 - 68 yrs. The Median age was 30 yrs. The number of males were 18. Number of females were 12.

Sites of involvement: Out of 30 cases of Synovial sarcoma, 21 cases $(70 \%)$ presented in lower limbs.5 cases in upper limbs. One case each was noted in aryepiglottic fold, lung, urinary bladder and mediastinum.

Clinical features: Most of the cases presented as painless swelling of the lower limbs. Pain was the presenting feature in 9 cases. Limping was seen in few cases. Pressure symptoms like numbness and dysphagia was noted in few cases.

Incidence of histological type: Out of total 30 cases of Synovial sarcoma17 (56.6\%) were Monophasic spindle type.

Grade $3+$ and $2+$ staining was taken as positive. Accordingly TLE 1 positivity was observed in 28/30 (93.3\%) cases of synovial sarcoma. The other tumours which showed positivity include $3 / 4$ (75\%) GIST cases, $1 / 2$ (50\%) Hemangiopericytoma, 4/4 (100\%) Schwannomas;1/1 Rhabdomyosarcoma (100\%). All cases of leiomyosarcoma, Ewing sarcoma, Dermatofibrosarcomaprotuberans, Fibrosarcoma, MPNST, Malignant fibrous histiocytoma and Solitary fibrous tumor included in the study were negative for TLE 1 .

Accordingly, TLE-1 showed a sensitivity of 93.3\%, specificity of $73.3 \%$ with positive predictive value of $77.77 \%$ and negative predictive value of $91.6 \%$.

Synovial sarcoma subtypes didn't show much difference in TLE 1 immunostaining. In case of biphasic tumor, TLE-1 positivity was noted in both epithelial and mesenchymal component. Intensity of TLE1 positivity was more in epithelial than mesenchymal component. Back ground staining is not observed in any of the tumor.

Table 1: Characterization features of synovial sarcoma and its histological mimics.

\begin{tabular}{|l|l|}
\hline Total number of cases $\mathrm{n}(\%)$ & Characterization features \\
\hline Leiomyosarcoma $\mathrm{n}=2(6.67 \%)$ & Classic morphology, with both Desmin and SMA positivity \\
\hline Gastrointestinal stromal tumour $\mathrm{n}=4(13.34 \%)$ & Classic morphology, with both CD117 and DOG1 positivity \\
\hline Hemangiopericytoma $\mathrm{n}=2(6.67 \%)$ & Classic morphology, CNS/PNS location and CD34 positivity \\
\hline Dermatofibrosarcoma protuberance $\mathrm{n}=3(10 \%)$ & Classic morphology and location \\
\hline Fibrosarcoma $\mathrm{n}=3(10 \%)$ & Classic morphology \\
\hline MPNST $\mathrm{n}=3(10 \%)$ & Classic morphology and S-100 positivity \\
\hline Schwannoma $\mathrm{n}=4(13.34 \%)$ & Classic morphology and S-100 positivity \\
\hline Solitary fibrous tumour $\mathrm{n}=2(6.67 \%)$ & Classic morphology and CD34+ \\
\hline Ewing sarcoma $\mathrm{n}=4(13.34 \%)$ & Classic morphology, CD99 Positivity. \\
\hline Rhabdomyosarcoma $n=1(3.34 \%)$ & Classic morphology and myogenin positivity. \\
\hline Desmoplastic small round cell tumor $\mathrm{n}=1(3.34 \%)$ & Classic morphology and IHC features. \\
\hline Malignant fibrous histiocytoma $n=1(3.34 \%)$ & Classic morphology and IHC features. \\
\hline
\end{tabular}


Table 2: Synovial sarcoma histopathological features.

\begin{tabular}{|l|c|c|}
\hline Tumour patterns & Positive $(\mathrm{n}=30)$ & \multicolumn{2}{|c|}{$73.3 \%$} \\
\hline Fascicles & 22 & $50 \%$ \\
\hline Hemangiopericytomatous & 15 & $40 \%$ \\
\hline Hyalinization & 12 & $33.3 \%$ \\
\hline Necrosis & 10 & $33.3 \%$ \\
\hline Calcification & 10 & $20 \%$ \\
\hline Myxoid changes & 6 & $20 \%$ \\
\hline Cystic degeneration & 5 & $16.6 \%$ \\
\hline Palisading & 5 & $10 \%$ \\
\hline Herringbone & 3 & $3.3 \%$ \\
\hline Ossification & 1 & \\
\hline
\end{tabular}

Table 3. IHC markers of Synovial sarcoma.

\begin{tabular}{|l|l|l|l|}
\hline IHC marker & Done $(\mathbf{n})$ & No. of Positive cases & \% Positivity \\
\hline BCL2 & 30 & 30 & $100 \%$ \\
\hline EMA & 28 & 26 & $92.8 \%$ \\
\hline CK & 25 & 17 & $68 \%$ \\
\hline S 100 & 8 & 2 & $25 \%$ \\
\hline CD 99 & 25 & 21 & $84 \%$ \\
\hline Vimentin & 12 & 10 & $83.3 \%$ \\
\hline DOG1 & 3 & - & $0 \%$ \\
\hline Desmin & 3 & - & $0 \%$ \\
\hline CD 34 & 5 & - & $0 \%$ \\
\hline Myogenin & 1 & - & $0 \%$ \\
\hline
\end{tabular}

Table 4: TLE1 expression in Synovial sarcoma and mimics.

\begin{tabular}{|c|c|c|c|c|}
\hline \multirow[t]{2}{*}{ Total number of cases $(n=60)$} & \multicolumn{4}{|c|}{ TLE1 Expression } \\
\hline & Grade 0 & Grade 1 & Grade 2 & Grade 3 \\
\hline Synovial sarcoma $(n=30)$ & 0 & 2 & 8 & 20 \\
\hline Leiomyosarcoma $(n=2)$ & 2 & 0 & 0 & 0 \\
\hline Gastrointestinal stromal tumour $(n=4)$ & 0 & 1 & 2 & 1 \\
\hline Ewing sarcoma $(n=4)$ & 4 & 0 & 0 & 0 \\
\hline Hemangiopericytoma $(n=2)$ & 1 & 0 & 1 & 0 \\
\hline DFSP $(n=3)$ & 3 & 0 & 0 & 0 \\
\hline Fibrosarcoma $(n=3)$ & 3 & 0 & 0 & 0 \\
\hline MPNST $(n=3)$ & 2 & 1 & 0 & 0 \\
\hline Schwannoma $(n=4)$ & 0 & 0 & 2 & 2 \\
\hline Solitary fibrous tumour $(n=2)$ & 1 & 1 & 0 & 0 \\
\hline Rhabdomyosarcoma $(n=1)$ & 0 & 0 & 1 & 0 \\
\hline Desmoplastic small round cell tumor(1) & 1 & 0 & 0 & 0 \\
\hline Malignant fibrous histiocytoma(1) & 0 & 1 & 0 & 0 \\
\hline
\end{tabular}


Table 5: TLE1 expression in synovial sarcoma.

\begin{tabular}{|c|c|c|c|c|}
\hline \multirow[t]{2}{*}{ Types of synovial sarcoma $(n=30)$} & \multicolumn{4}{|c|}{ TLE1 Expression } \\
\hline & Grade 0 & Grade 1 & Grade 2 & Grade 3 \\
\hline Monophasic SS(19) & 0 & 1 & 5 & 13 \\
\hline Biphasic SS (5) & 0 & 1 & 1 & 3 \\
\hline Poorly differentiated SS (4) & 0 & 0 & 2 & 2 \\
\hline Calcifying type of SS (2) & 0 & 0 & 1 & 1 \\
\hline
\end{tabular}

Table 6: Expression of TLE1 marker in SS mimics ( Differential diagnosis).

\begin{tabular}{|c|c|c|c|c|c|}
\hline Histological type & $\begin{array}{l}\text { Total number of } \\
\text { cases }\end{array}$ & TLE1 grade 0 & TLE1grade1 & TLE1 grade2 & TLE1 grade3 \\
\hline LMS & 2 & 2 & 0 & 0 & 0 \\
\hline GIST & 4 & 1 & 2 & 1 & 0 \\
\hline DFSP & 1 & 1 & 0 & 0 & 0 \\
\hline FS & 3 & 2 & 1 & 0 & 0 \\
\hline SFT & 2 & 2 & 0 & 0 & 0 \\
\hline Schwannoma & 4 & 1 & 1 & 2 & 0 \\
\hline MPNST & 3 & 2 & 1 & 0 & 0 \\
\hline HPC & 1 & 1 & 0 & 0 & 0 \\
\hline ES(PDSSmimics) & 4 & 0 & 1 & 3 & 0 \\
\hline MCH(PDSS mimics) & 1 & 1 & 0 & 0 & 0 \\
\hline
\end{tabular}

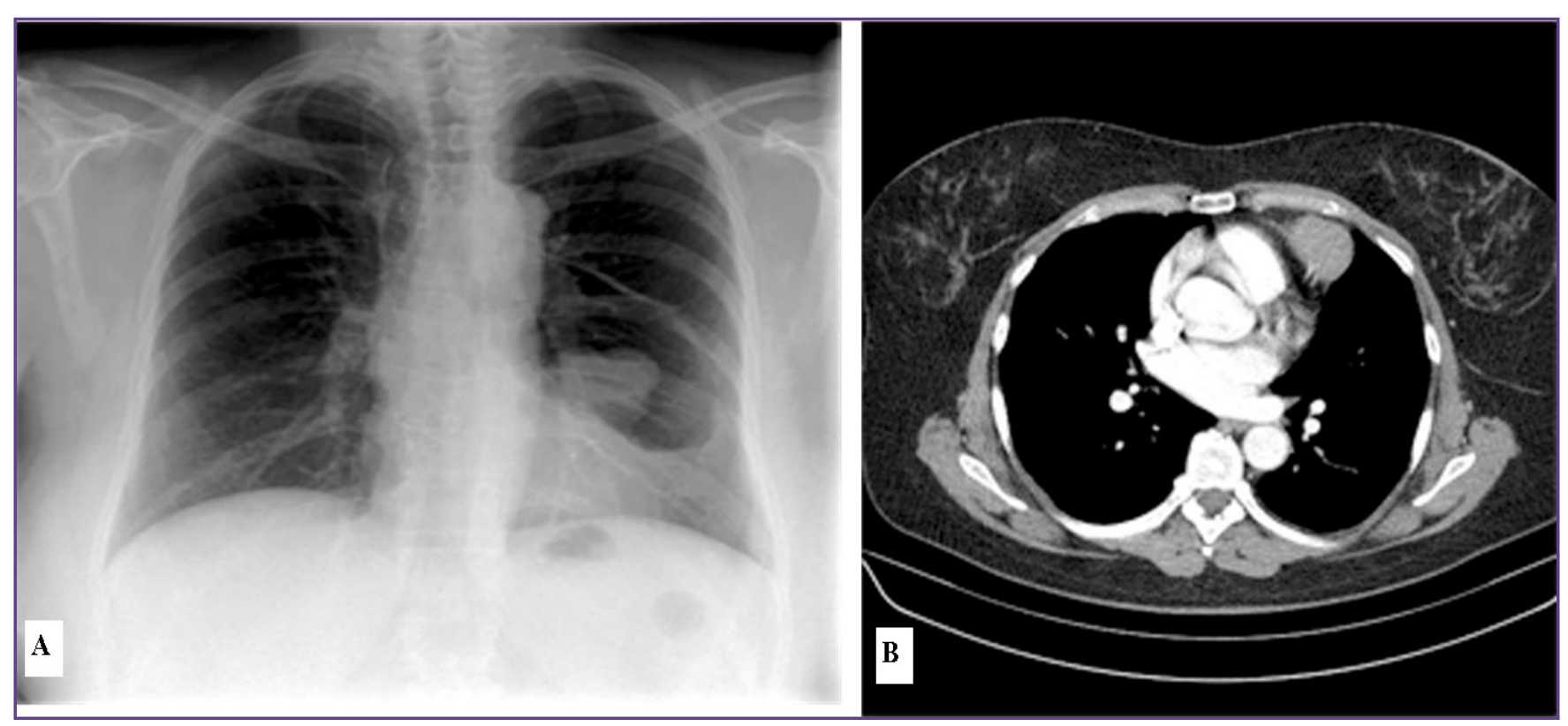

Fig. 1: A. Chest radiograph- Mass in the left mid zone of lung. B. Chest computed tomography showed an $3 \times 5 \mathrm{~cm}$ measuring intrathoracictumor with broadly based contact to the pericardium, surrounded by pneumatocele. 

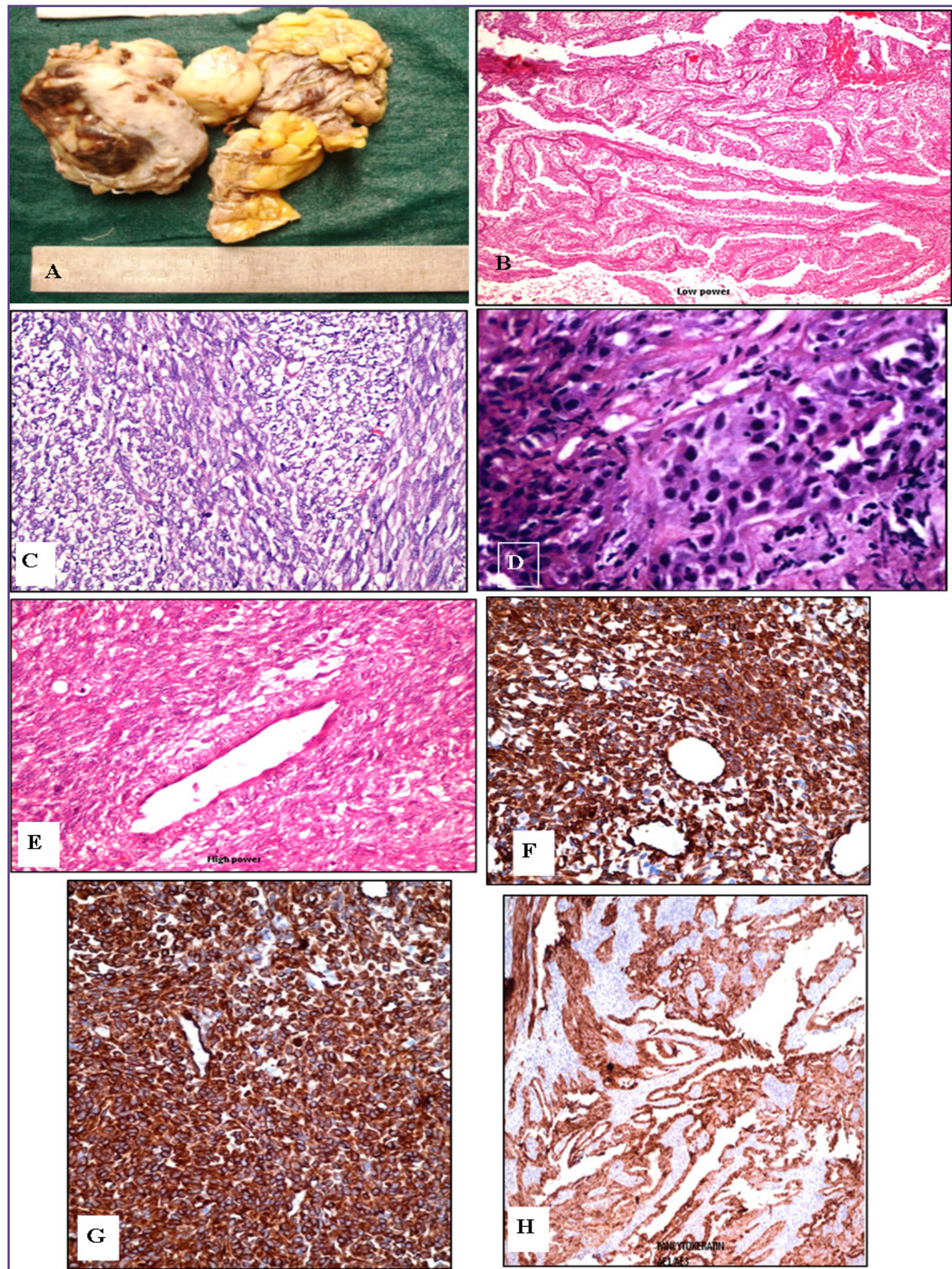

Fig. 2: Macroscopic images of Synovial sarcoma A. Biopsy of Synovial sarcoma. B. Haemotoxylin and Eosin staining shows Biphasic Synovial sarcoma (10x)). C. Staining shows Monophasic spindle Synovial sarcoma (40x). D. Staining shows Calcifying variant (40x). E. Staining shows Peritheliomatous pattern in Synovial sarcoma Synovial sarcoma. F. Immunohistochemistry of Synovial sarcoma by CD 99 Positive. G. Immunohistochemistry of Synovial sarcoma-EMA Positive. H. Immunohistochemistry Synovial sarcoma-CK positive. 

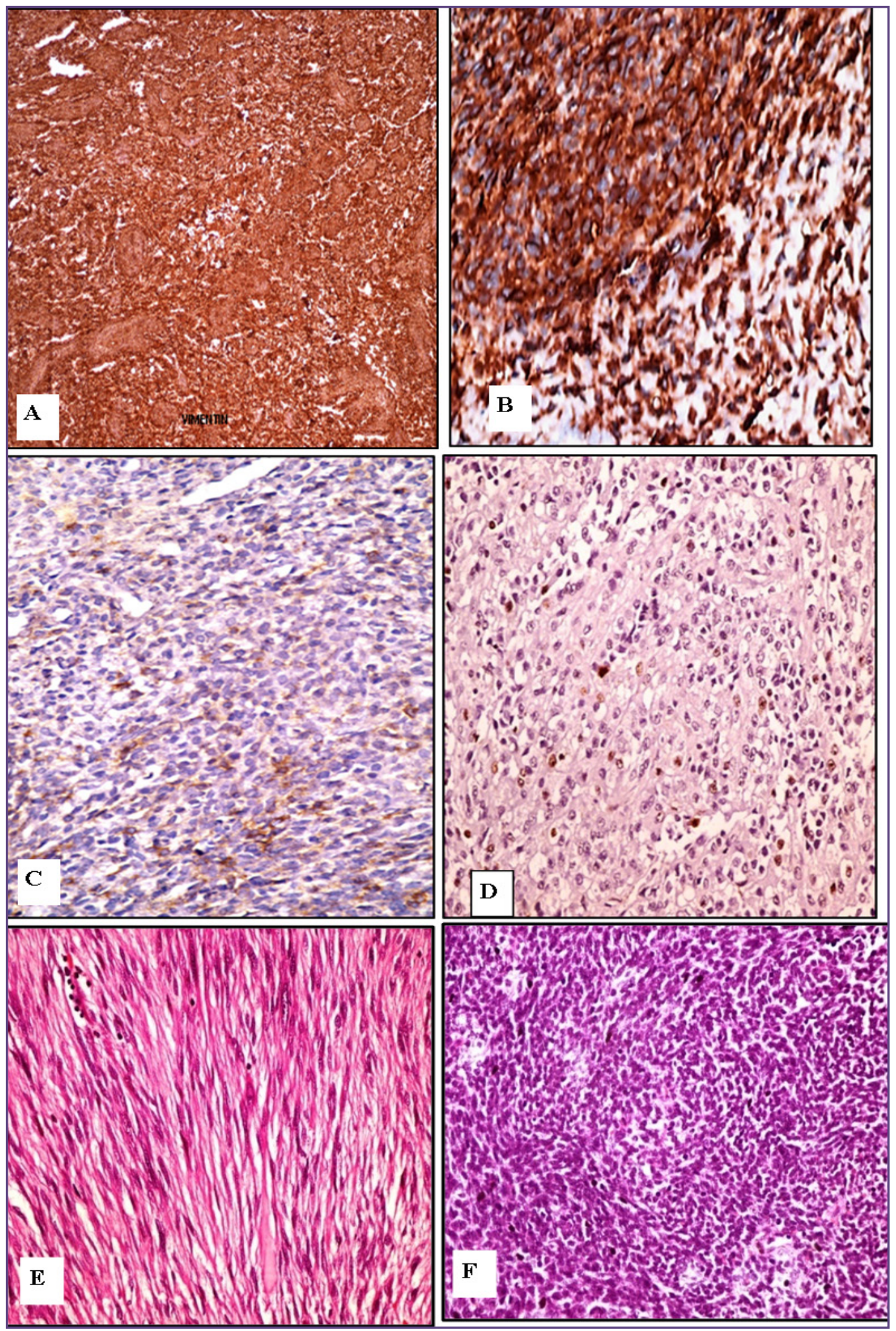

Fig. 3. A. Immunohistochemistry markers of Synovial sarcoma Vimentin positive. B. Immunohistochemistry markers of Synovial sarcoma-Bcl2 positive . C. Desmin positive . D. Ki 67 low index. E. Malignant GIST F. Rhabdomyosarcoma. 

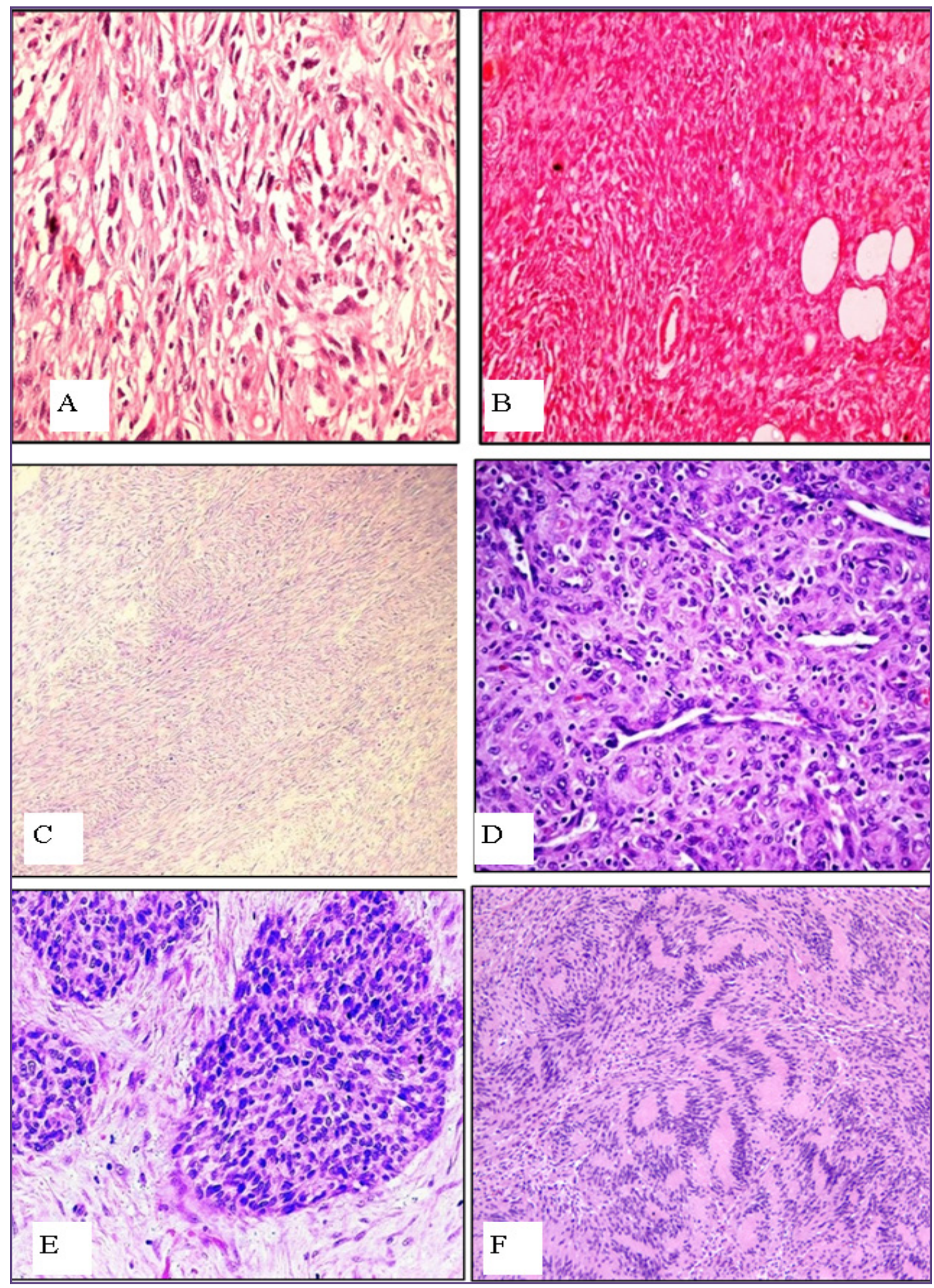

Fig. 4: A. Leiomyosarcoma. B. Dermatofibrosarcoma protuberans (DFSP). C. Fibrosarcoma. D. Infantile hemangiopericytoma. E. Desmoplastic small round cell tumor. F.Schwannoma. 

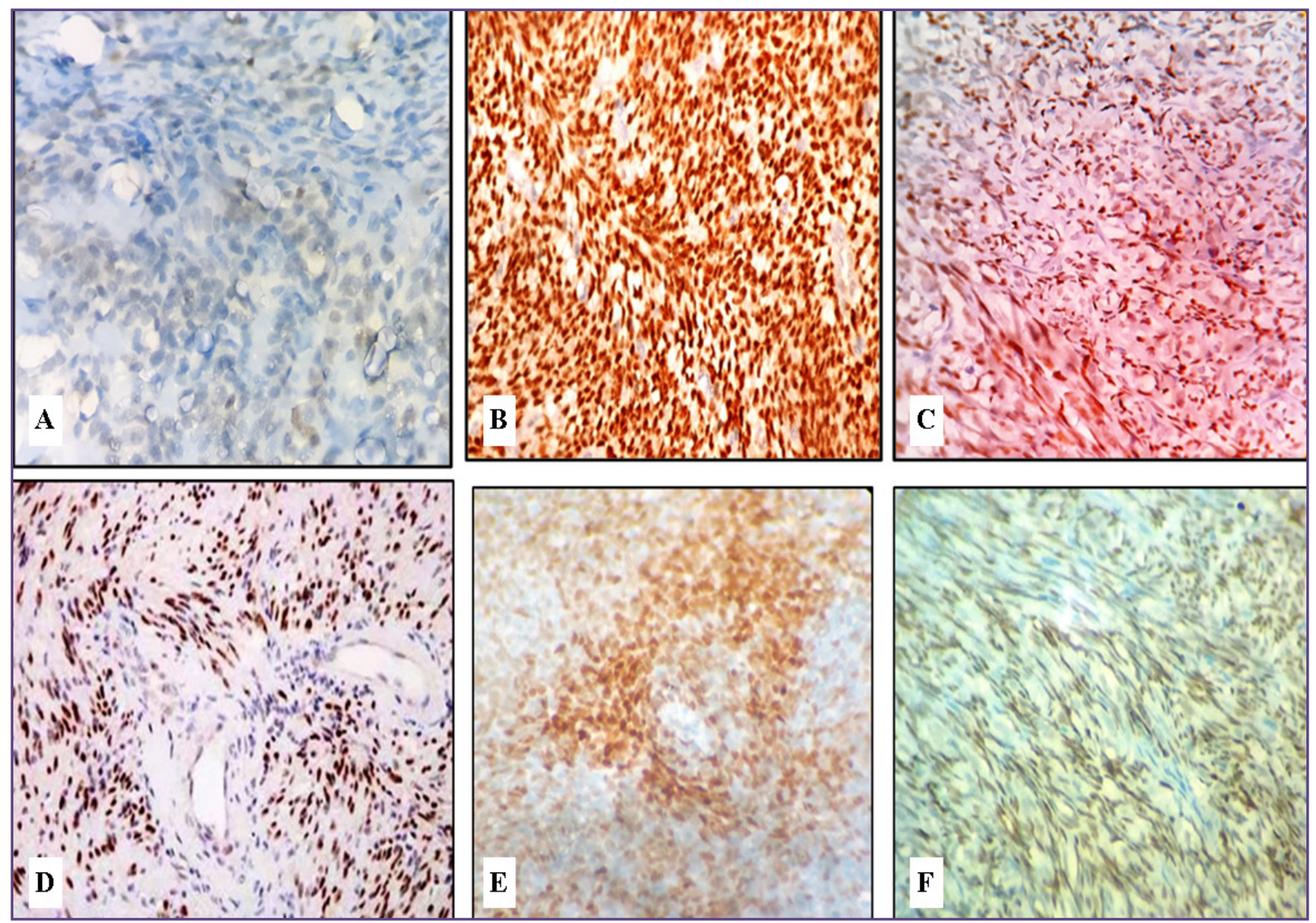

Fig. 5: Monophasic spindle grade 1. B. Monophasicspindle grade 3. C. Biphasic SS grade 3. D. Schwannoma grade 3. E. Rhabdomyosarcomagrade 3. F. Search Results-Gastrointestinal stromal tumor GIST grade 3.

\section{Discussion}

The World Health Organization (WHO) has defined synovial sarcomas (SS) as a"mesenchymal spindle cell tumors which display variable epithelial differentiation, including glandular formation and have a specific chromosomal translocation $\mathrm{t}(\mathrm{x}: 18)(\mathrm{p} 11 ; \mathrm{q} 11)$ and that leads to formation of a SS18-SSX fusion gene".

In the present study the age range was between 10-68 years with a median age of 30 years. The present study was in comparision with Okcuet et al. and Siegel et al, in showing male preponderance. ${ }^{[9,10]}$

In the present study the tumor size varied from trucut biopsies of synovial sarcomas to mass measuring $10 \times 10 \mathrm{cms}$ with majority of cases presenting as painless swelling.

In the present study, most common mode of presentation was painless swelling. Around 53\% cases presented with painless swelling and $30 \%$ of cases presented with painful swelling.
In the Present study, Swelling was seen in 53\% of cases, Pain in $30 \%$, Limping $23 \%$, Joint effusion was observed in $17 \%$, Numbness in $07 \%$ and Dysphagia in $07 \%$ ). Joint effusion, numbness and dysphagia were because of pressure symptoms due to compression by large mass.

In the present study lower limbs were the most common sites of involvement followed by upper limbs. One case was noted in lung, mediastinum, aryepiglottic fold and urinary bladder. Involvement of aryepiglottic fold was seen in present study, where as it was not found in any other studies. Involvement of lower limbs is the most common site of involvement in present study, and was in comparision with recent studies. ${ }^{[1-13]}$

Synovial sarcomascomprises a morphologically heterogenous group, that includes biphasic, monophasic spindle, monophasic epithelial, poorly differentiated and calcifying types. In the present study, commonest histological type was monophasic spindle with 19 cases 
(56\%), biphasic 5 cases (20\%), monophasic epithelial 2 cases $(8 \%)$, poorly differentiated 4 cases $(8 \%)$, calcifying variant 2 cases $(8 \%)$.

Monophasic spindle was the most common histological type in the present study and was in comparision with other studies which also observed that monophasic spindle as the most common variant.

In the present study there were 4 cases of poorly differentiated SS. Foo et al. ${ }^{[7]}$ described 22 cases of poorly differentiated SS. Other also shows similar differentiation. [14]

Calcifying variant of SS was described only in the present study. Calcifying SS was not described in other studies. CD99 positivity was seen in $84 \%$ of cases in present study, whereas it was $70-75 \%$ positivity in Okcu et al. and $74.1 \%$ in Rekhi et al. study. ${ }^{[8,9]}$

EMA positivity was observed in $92.8 \%$ of cases in present study, whereas it showed $80-100 \%$ positivity in Okcu et al. and $764 \%$ Rekhi et al study. Vimentin positivity was observed in $83.3 \%$ of cases in present study. Desmin positivity was not seen in present study, whereas Okcu et al observed $30 \%$ positivity.

Bcl2 showed $100 \%$ positivity in present study, this finding was in comparison with other studies. ${ }^{[15]}$

Synovial sarcoma has wide anatomic distribution and variable histological patterns, which create diagnostic difficulties. Monophasic fibrous synovial sarcoma can be difficult to distinguish from its histological mimics which include other spindle cell sarcomas such as Malignant peripheral nerve sheath tumours, Cellular Schwannomas, Solitary fibrous tumour, Fibrosarcoma, DFSP, Hemangiopericytoma, Malignant fibrous histiocytoma, GIST and Leiomyosarcoma. Mimics of poorly differentiated synovial sarcoma include Ewings sarcoma, Desmoplastic small round cell tumor and Rhabdomyosarcoma. Though IHC is helpful in differentiation, in many cases there may be overlapping immunohistochemical results. Molecular analysis is gold standard for diagnosis of synovial sarcoma, but use of these ancillary techniques is limited by many practical issues like cost and limited resources. Many attempts have been made to identify highly specific, sensitive marker for SS. Gene expression studies showed over expression of TLE family genes, particularly TLE 1 in SS.

In the present study, sensitivity and specificity of TLE 1 for SS is $93.3 \%$ and $73.3 \%$ respectively which is similar to several other studies. ${ }^{[16]}$ Studies by Kosemehmetoglu et $\mathrm{al}^{\left[{ }^{[17]}\right.}$ and Foo et $\mathrm{a}^{\left[{ }^{[7]}\right.}$ showed lesser sensitivity. However all these studies employed different grading scheme to asses TLE1 positivity. In the present study the number of cases with grade $3+(19 / 30 ; 64 \%)$ positivity was higher than grade $2+$ positive cases $(9 / 30 ; 30 \%)$ which is similar to that reported by Terry et al. and Rekhi et al .The remaining two TLE1 negative cases of SS in the present series was one MSS and one biphasic and both showed grade 1staining. Though these 2 cases had classical morphology and IHC findings of SS, molecular confirmation was not done.

One of the limitations of present study compared to others was inclusion criteria for SS, which was mainly based on morphology and IHC findings, while others included molecularly confirmed cases. We tried to overcome this, by rigid morphological and IHC criteria. The correlation of present study results with that of other studies, further strengthens our inclusion criteria. The present study and that of Terry et al. ${ }^{[18]}$ was carried out on tissue microarray, whereas the other studies used whole sections, however the results were well correlated. There was no significant difference in sensitivity for TLE1 among various histological types of SS included in present study, whereas Foo et al. observed higher sensitivity for PDSS (91\%) when compared to overall sensitivity of SS $(82 \%)$. The analysis of SS mimics showed a variation in the entities as well as number of cases included in different studies to analyze specificity of TLE 1.The positivity of MPNST to TLE 1 reported in previous studies ranges from 2.3-30\%. In the present study all 3 were negative. Schwannomas have also shown wide range of positivity ranging from 0 $100 \%$. In present study, there was $100 \%$ (4/4) positivity. TLE1 on GIST was done in two studies- Kosemehmetoglu et al. and Rekhi et al. in 6 and 1 cases respectively and were negative whereas Terry et al studied 35 cases and showed positivity in $6 \%$ of their cases. The present study included 4 cases of GIST, of which 3 were positive \& 1 negative for TLE1 and all 4 were CD117 and DOG1 positive thereby concluding them as GIST rather than SS. There were 4 cases of Ewings sarcoma included in the present study which were TLE1negative. Similar results were reported by many other studies as well, except for the study by Rekhi et al, who demonstrated positivity in $40 \%$ of their cases. This could be due to different sensitivity of the antibody clone used. Of the 2 Hemangiopericytomas in the present study $1 / 2$ cases $(50 \%)$ had TLE1 positivity whereas Terry et al observed $40 \%$ positivity and is the only previous study to analyse TLE1immunostaining in Hemangiopericytomas. The reported TLE1 positivity in leiomyosarcoma has been low ranging from $2-20 \%$. In present study, all the 2 cases were negative. All cases of 
Dermatofibrosarcoma protuberance and Fibrosarcoma were negative for TLE 1 in the present study as was seen in other studies. Analysis of TLE1 on rhabdomyosarcoma was done in few previous studies. The reported TLE1 positivity in Rhabdomyosarcoma ranged from $0-100 \%$. In present study the single case was in comparision with Kosemehmetoglu and Rekhi et al. TLE1 on desmoplastic small round cell tumor was done in few previous studies. The reported TLE1 positivity in desmoplastic small round cell tumor was $0 \%$. Analysis of TLE 1 on malignant fibrous histiocytoma was negative in literature, similar to single case in present study. ${ }^{[8,14,17]}$

The present study showed that most of the tumors (SS mimics) other than SS showed less intensity of positivity to TLE1-Refer table 9\&11.Chaung et al also observed the same and concluded that, grading could differentiate SS from its mimics Negative predictive value of TLE 1 is $100 \%$ in study done by Jagdis et al. ${ }^{[19]}$. In the present study Negative Predictive Value is $91.6 \%$ which is close to the previous study whereas the Positive Predictive Value in this study is $77.7 \%$ which is much less when compared to Jagdis et al (92\%) study.

The other limitation of present study was follow up was not available for all cases. In the present study Negative Predictive Value is $91.6 \%$ which is close to the previous study whereas the Positive Predictive Value in this study is $77.7 \%$ which is much less when compared to jagdis et al (92\%) study. ${ }^{[19]}$

\section{Conclusion}

Immunohistochemistry studies for TLE1 in differential diagnosis, to molecular genetic testing is limited. Small round blue cell tumors should be kept in mind while diagnosing poorly differentiated SS. In our study, TLE1 Immunohistochemistry showed sensitivity of $93.3 \%$, specificity of $73.3 \%$ with positive predictive value of $77.77 \%$ and negative predictive value of $91.6 \%$. Less specificity because of its positive expression in other mesenchymal tumors.

\section{References}

1. Dimitriadis E, Rontogianni D, Kyriazoglou A, Takou A, Frangia K, Pandis N, et al. Novel SYT-SSX fusion transcript variants in synovial sarcoma. Cancer Genet Cytogenet. 2009;195(1):54-8.

2. Fletcher, Christopher DM, K. Krishnan Unni, and Fredrik Mertens, eds. Pathology and genetics of tumours of soft tissue and bone. Vol. 4. Chapter ix. IARC Press: Lyon, 2002. page: $200-204$.

3. Baek D, Barbieri A, Ayala AG, Lee KM, Ju MJ, Ro JY. Monophasic epithelial synovial sarcoma initially diagnosed as metastatic adenocarcinoma of unknown primary. Human Pathology: Case Reports. 2019;17: 200307.

4. Thway K, Fisher C. Synovial sarcoma: defining features and diagnostic evolution. Ann Diagn Pathol. 2014;18(6):369-80.

5. Pop DL, Folescu R, Deleanu BN, Iacob M, VERMEŞ Prejbeanu R, et al. The role of immunohistochemistry in the diagnosis and management of synovial sarcoma. Rom J Morphol Embryol. 2018;59(2):569-72.

6. Shooshtarizadeh P, Helness A, Vadnais C, Brouwer N, Beauchemin H, Chen R, Bagci H, Staal FJ, Coté JF, Möröy $\mathrm{T}$. Gfilb regulates the level of $\mathrm{Wnt} / \beta$-catenin signaling in hematopoietic stem cells and megakaryocytes. Nat Commun. 2019;10(1):1-6.

7. Foo WC, Cruise MW, Wick MR, Hornick JL. Immunohistochemical staining for TLE1 distinguishes synovial sarcoma from histologic mimics. Am J Clin Pathol. 2011;135(6):839-44.

8. Rekhi B, Basak R, Desai SB, Jambhekar NA. Immunohistochemical validation of TLE1, a novel marker, for synovial sarcomas. Indian J Med Res. 2012;136 (5):766-75.

9. Okcu MF, Munsell M, Treuner J, et al. Synovial sarcoma of childhood and adolescence: a multicenter, multivariate analysis of outcome. J Clin Oncol. 2003;21:1602-1611.

10. Segal NH, Pavlidis P, Antonescu CR, et al. Classification and subtype sprediction of adult soft tissue sarcoma by functional genomics. Am J Pathol. 2003;163:691-700.

11. Al-Mohrej OA, Al-Jarallah SA, Al-Dakhil Allah HH, Pant $\mathrm{R}, \mathrm{Al}$-Zayed ZS. Synovial sarcoma presenting as an intraarticular mass in a pediatric patient: a case report. BMC Musculoskelet Disord 2020;21: 21, 1-5.

12. Chhabra A, Ashikyan O, Slepicka C, Dettori N, Hwang H, Callan A, et al. Conventional MR and diffusion-weighted imaging of musculoskeletal soft tissue malignancy: correlation with histologic grading. Eur Radiol. 2019;29:4485-94.

13. Rose L, Grignon D, Cheng L, Fan R, Zhang S, Alruwaii F, Chen S. Primary renal synovial Sarcomas: PAX 8 immunostaining and unusual molecular findings. APPL IMMUNOHISTO M M. 2020;28(3):221-8.

14. Rekhi B, Shetty O, Ramadwar M, Rangarajan V, Bajpai J. Role of fine needle aspiration cytology in the diagnosis of a rare case of a poorly differentiated synovial sarcoma with "Rhabdoid" features, including treatment implications. Diagn Cytopathol. 2017;45(7):662-7.

15. Argani P, Faria PA, Epstein JI, Reuter VE, Perlman EJ, Beckwith JB, et al. Primary renal synovial sarcoma: molecular and morphologic delineation of an entity previously included among embryonal sarcomas of the kidney. Am J Surg Pathol. 2000;24(8):1087-96.

16. Pukhalskaya T, Smoller BR. TLE1 expression fails to distinguish between synovial sarcoma, atypical fibroxanthoma, and dermatofibrosarcoma protuberans. J. Cutan. Pathol. 2020;47(2):135-8. 
17. Kosemehmetoglu K, Vrana JA, Folpe AL. TLE1 expression is not specific for synovial sarcoma: a whole section study of 163 soft tissue and bone neoplasms. Mod Pathol. 2009;22(7):872-8.

18. Terry J, Saito T, Subramanian S, Ruttan C, Antonescu CR, Goldblum JR, et al. TLE1 as a diagnostic immunohistochemical marker for synovial sarcoma emerging from gene expression profiling studies. Am J Surg Pathol. 2007;31(2):240-6.

19. Jagdis A, Rubin BP, Tubbs RR, Pacheco M, Nielsen TO. Prospective evaluation of TLE1 as a diagnostic immunohistochemical marker in synovial sarcoma. Am J Surg Pathol. 2009;33(12):1743-51.

*Corresponding author:

Dr. Sujitha Chougani, Consultant Pathologist, Yashoda Hospital, Secunderabad, Telangana, INDIA

Email: suji.renukadevi@gmail.com

Date of Submission : 25/01/2020

Date of Acceptance : 07/07/2020

Financial or other Competing Interests: None.
Date of Publication : 30/08/2020 